\title{
A kinetic core model of the glucose-stimulated insulin secretion network of pancreatic $\beta$ cells
}

\author{
Nan Jiang $\cdot$ Roger D. Cox $\cdot$ John M. Hancock
}

Received: 15 December 2006/ Accepted: 2 March 2007/Published online: 21 May 2007

(C) Springer Science+Business Media, LLC 2007

\begin{abstract}
The construction and characterization of a core kinetic model of the glucose-stimulated insulin secretion system (GSIS) in pancreatic $\beta$ cells is described. The model consists of 44 enzymatic reactions, 59 metabolic state variables, and 272 parameters. It integrates five subsystems: glycolysis, the TCA cycle, the respiratory chain, $\mathrm{NADH}$ shuttles, and the pyruvate cycle. It also takes into account compartmentalization of the reactions in the cytoplasm and mitochondrial matrix. The model shows expected behavior in its outputs, including the response of ATP production to starting glucose concentration and the induction of oscillations of metabolite concentrations in the glycolytic pathway and in ATP and ADP concentrations. Identification of choke points and parameter sensitivity analysis indicate that the glycolytic pathway, and to a lesser extent the TCA cycle, are critical to the proper behavior of the system, while parameters in other components such as the respiratory chain are less critical. Notably, however, sensitivity analysis identifies the first reactions of nonglycolytic pathways as being important for the behavior of the system. The model is robust to deletion of malic enzyme activity, which is absent in mouse pancreatic $\beta$ cells. The model represents a step toward the construction of a model with species-specific parameters
\end{abstract}

Electronic supplementary material The online version of this article (doi:10.1007/s00335-007-9011-y) contains supplementary material, which is available to authorized users.

N. Jiang · J. M. Hancock $(\bowtie)$

Bioinformatics Group, MRC Mammalian Genetics Unit,

Harwell, Oxfordshire OX11 0RD, UK

e-mail: j.hancock@har.mrc.ac.uk

R. D. Cox

Type 2 Diabetes Group, MRC Mammalian Genetics Unit,

Harwell, Oxfordshire OX11 0RD, UK that can be used to understand mouse models of diabetes and the relationship of these mouse models to the human disease state.

\section{Introduction}

Type 2 diabetes mellitus (T2DM), along with associated problems such as hypertension, dyslipidemias, and obesity, is an increasing problem in human populations: 150 million individuals are currently affected and a rapid expansion is expected during the next 20 years (Freeman and Cox 2006). Human genetic studies provide strong evidence that predisposition to T2DM, and in particular its defining phenotype, glucose intolerance, has a complex genetic basis (McCarthy 2004). This is supported by studies in animal models. A significant number of candidate genes for involvement in predisposition to T2DM are implicated in pancreatic $\beta$-cell dysfunction (Freeman and Cox 2006). Glucose-stimulated insulin secretion (GSIS) is the pivotal homeostatic process in the control of blood glucose levels and takes place in pancreatic $\beta$ cells (Ashcroft and Rorsman 2004). GSIS takes in a number of relatively well characterized biochemical pathways such as glycolysis, the TCA cycle, and the respiratory chain (Fig. 1A), but new discoveries implicate novel features of $\beta$-cell biochemistry (Eto et al. 1999; Newgard et al. 2002; Ronnebaum et al. 2006; Rubi et al. 2004). The effects of individual mutations on the GSIS system are not always intuitively obvious and qualitative descriptions do not allow for a quantitative analysis of these effects. It should be possible to overcome these problems by building explicit mathematical models of the system.

Systems biology aims at system-level understanding of biological processes and how a system's behavior emerges 


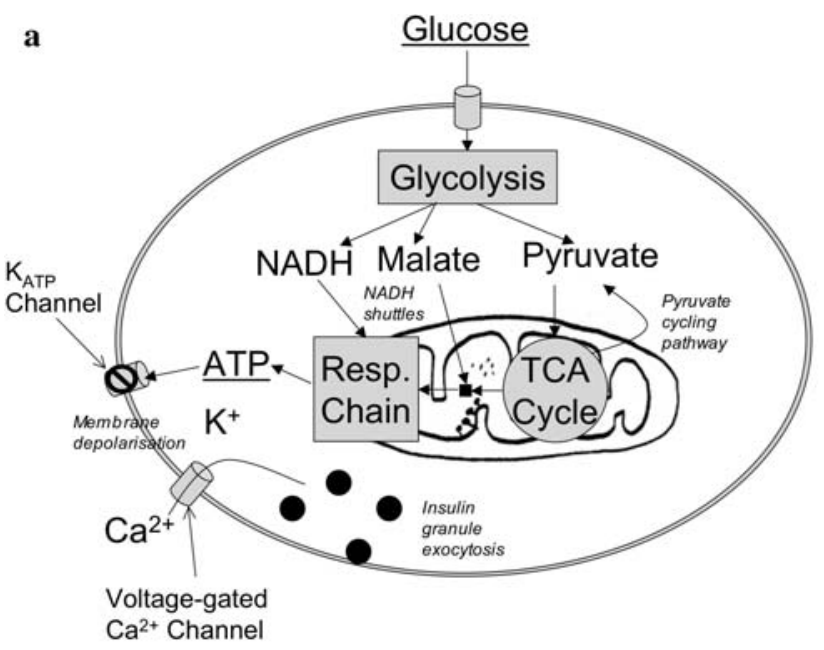

b

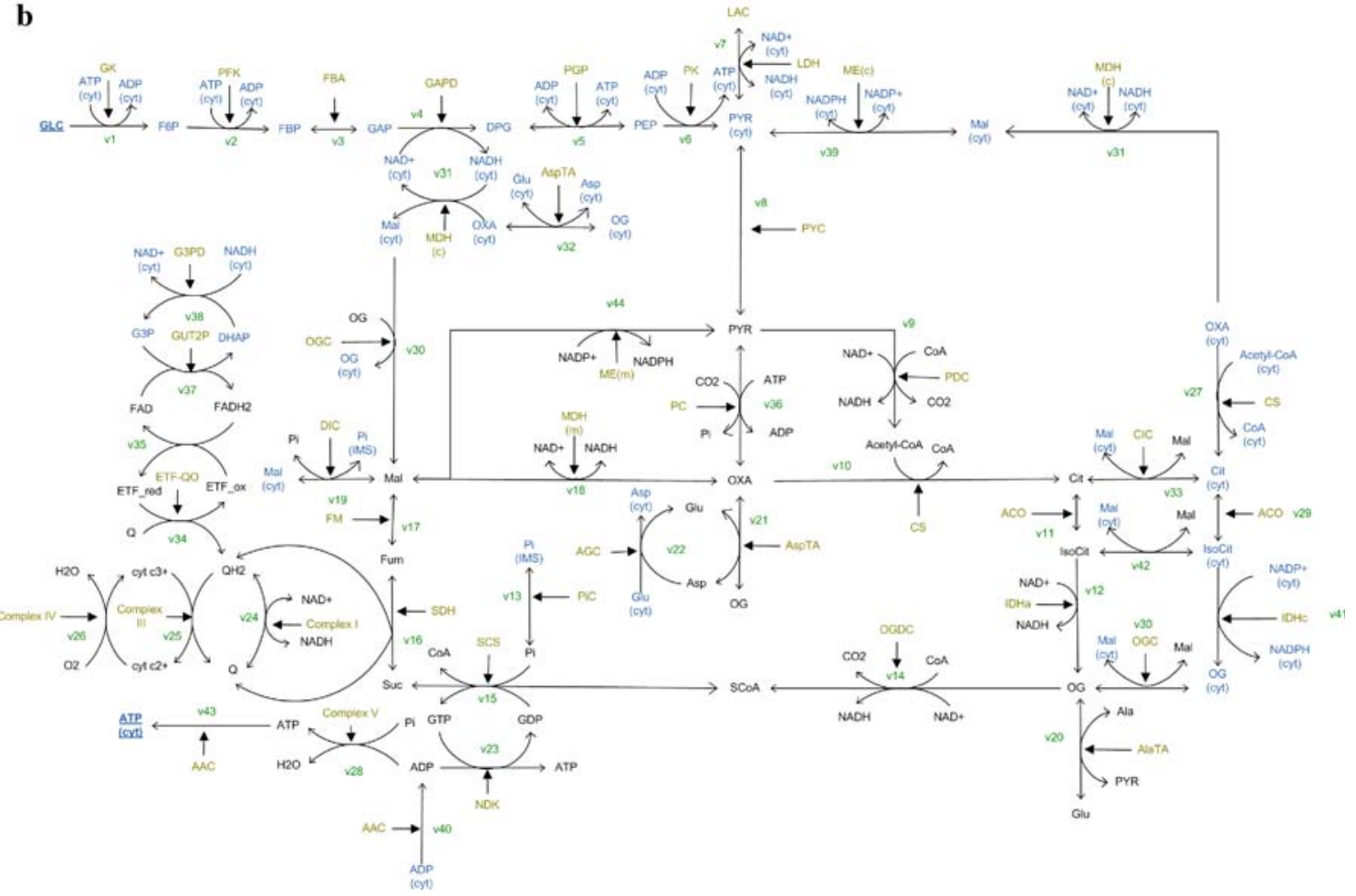

Fig. 1 Pathways involved in GSIS. A Diagrammatic representation of processes linking glucose uptake to insulin secretion in pancreatic $\beta$ cells. The diagram represents processes taking place in the presence of extracellular glucose. Glucose is imported into the $\beta$ cell and undergoes glycolysis. The primary product of glycolysis is pyruvate, which is imported into mitochondria. Malate, an intermediate product of glycolysis and NADH, also a product of this pathway, may also be imported into mitochondria via the NADH shuttles. Malate is converted to fumarate, which is also a product of the TCA cycle fed by pyruvate and is a substrate of the respiratory chain, whose end product is ATP. Imported NADH can also feed into the respiratory chain. Pyruvate may also be recycled from the mitochondrial matrix

from the interactions among its components. An objective milestone for successful cell simulation might be the construction of a whole metabolic model. Consequently, biochemical dynamic models composed of a relatively large to the cytoplasm via the pyruvate cycling pathway. ATP interacts with the $\mathrm{K}_{\mathrm{ATP}}$ channel to block it, resulting in an increase in intracellular $\mathrm{K}^{+}$and depolarization of the cell membrane. This in turn leads to activation of the voltage-gated $\mathrm{Ca}^{2+}$ channel, influx of $\mathrm{Ca}^{2+}$, and activation of insulin granule exocytosis. B Overview of the kinetic network modeled. Reactions are represented as arrows (which may be uni- or bidirectional) and labeled v1 to v44. Metabolites, which are transformed by the reactions, are labeled according to their cellular location: metabolites in the cytoplasm and intermembrane space are labeled in blue, and metabolites in the mitochondrial matrix are labeled in black. Enzymes catalyzing reactions are labeled in brown. Abbreviations are as in Table 1

number of metabolic reactions are being developed. Examples are models of central carbon metabolism in E. coli (Chassagnole et al. 2002; Varner 2000), glycolysis in lactic acid bacteria (Hoefnagel et al. 2002), mitochondrial 
NADH shuttles and anaplerosis in $\beta$ cells (Westermark et al. 2006), and mitochondrial ATP production (Bertram et al. 2006; Magnus and Keizer 1997, 1998a, 1998b). The advantage of such detailed, biochemically formulated models is that a one-to-one comparison can be made between model and experiment. Thus, they provide platforms that allow discovery of new intrinsic biological properties.

A number of mathematical and computational models have been developed related to the GSIS system. Topp et al. (2000) designed a model that includes three ordinary differential equations (ODEs) representing the dynamics of glucose and insulin within the mass of $\beta$ cells. As a coarsegrid model, the model concentrates on investigating the normal behavior of the glucose regulatory system and pathways into diabetes. Another minimal model was developed by Bertram et al. (2006). They built a simplified ATP synthesis model based on earlier models of oxidative phosphorylation (Magnus and Keizer 1997, 1998a, 1998b) to capture the same behavior as in Magnus and Keizer's models. In the Bertram et al. model, they took pyruvate as the main input of their system and ATP as the end product. Four mitochondrial variables $\left(\mathrm{NADH}_{\mathrm{m}}, \mathrm{ADP}_{\mathrm{m}}, \Delta \Psi\right.$, and $\mathrm{Ca}_{\mathrm{m}}$ ) are described with equations corresponding to the dynamics of different types of fluxes or reactions. With their simplified model they investigated the dynamics of the four mitochondrial variables versus the change of glycolytic flux and pulses of calcium. A more refined simulation model for the mitochondrial system was developed by Yugi and Tomita (2004). In this model 58 enzymatic reactions and 117 metabolites are included to represent four pathways (respiratory chain, the TCA cycle, fatty acid $\beta$ oxidation, and the inner-membrane transport system) in mitochondria. Previously published enzyme kinetics studies from the literature were integrated into a single dynamic model using the E-cell2 simulation platform (http://www.e-cell.org).

Following recent research on the importance of NADH shuttles in ATP production, a mathematical model of mitochondrial NADH shuttles has been developed by Westermark et al. (2006). The model comprises the mitochondrial NADH shuttles and mitochondrial metabolism, which in total included 19 enzyme reactions and 10 metabolites with simplifications on the boundary of the system. The model reproduces the experimental finding by Eto et al. (1999) that blocking the NADH shuttles attenuates the signaling to ATP production while retaining the rate of glucose oxidation. Besides the model of NADH shuttles, Westermark and Lansner (2003) also constructed a model of the upper part of glycolysis in the pancreatic $\beta$ cell. The model concentrated on studies on the enzymatic reactions of PFK (phosphofructokinase), which has been shown to control metabolite oscillations in the glycolysis pathway. Although more and more computational dynamic models have been constructed, a complete, detailed kinetic model with a large number of enzyme rate equations mimicking the dynamics from glucose input to ATP production has not been developed so far.

We therefore initiated the development of a quantitative, kinetic model of the core processes in GSIS as an aid to understanding genetic and biochemical data in mouse models of T2DM. Constructing such a model poses a significant technical and intellectual challenge because the GSIS system consists of at least five biochemical subpathways (glycolysis, TCA cycle, the respiratory chain, NADH shuttles, and the pyruvate cycle) that take place in more than one cellular compartment (cytoplasm and mitochondrion). In addition, the system consists of a large number of interacting metabolites and enzymes. Here we describe a kinetic model of the pathways leading from the beginning of glycolysis to ATP production, which we call the core process. We show that the model has qualitative properties consistent with expectations, including showing oscillations in the glycolysis pathway and in ATP concentration. We also discuss some analyses of the properties of the network and its applicability to mouse models and the understanding of human disease.

\section{Methods}

We used mathematical modeling in which all metabolite interactions are described in terms of kinetic equations. The components of our mathematical model are kinetic parameters and state variables, which indicate the state of a system at a certain time. The kinetic parameters include Michaelis-Menten constants, rate constants of association and dissociation, etc. Most of the kinetic parameters were taken from the literature (see Supplementary Materials).

Our model is based on ODEs and consists of 44 enzymatic reactions, 59 metabolic state variables, and 272 parameters. The number of parameters is large because most of the rate equations inside the mitochondrion follow complex reaction mechanisms (Ping-pong $\mathrm{Bi} \mathrm{Bi}$, Ordered $\mathrm{Bi} \mathrm{Bi}$, etc.), although identical reactions in different compartments have been assumed to have the same parameters. The following is a representative derivation of one of the 59 ODEs. To determine the change in the concentration of a certain metabolite $\left[m_{i}\right]$ over time, we calculate the sum of the reaction rates producing $\left[m_{i}\right]$ minus the rates consuming $\left[m_{i}\right]$. Thus, we get the following differential equation, where $i$ represents one of the 59 metabolites:

$\frac{d\left[m_{i}\right]}{d t}=\sum v_{\text {production }}-\sum v_{\text {consumption }}$

The simulation is then equivalent to solving the following differential equations: 
$\frac{d\left[m_{i}\right]}{d t}=f\left(t,\left[m_{i}\right], p\right)$

$m\left(t_{0}\right)=m_{0}(p)$

in which $p$ represents the parameters in the model.

\section{Constructing the network}

There is a growing appreciation of the complexity of the pathways involved in GSIS from pancreatic $\beta$ cells. Traditionally, it has been accepted that an increase in the ATP/ ADP ratio brought about by glucose flux through glycolysis and the tricarboxylic acid (TCA) cycle is central to the GSIS. In addition, it has been demonstrated that the contributions of the TCA cycle and NADH shuttle system to mitochondrial ATP production may be approximately equal (Eto et al. 1999; Rubi et al. 2004). Recently, the role of cytosolic NADP-dependent isocitrate dehydrogenase (ICDc) in control of GSIS in $\beta$ cells has been studied (Newgard et al. 2002; Ronnebaum et al. 2006). From these studies it has been concluded that the pyruvate cycling pathway involving ICDc plays an important role in the control of GSIS. Therefore, in our model we included five subpathways: glycolysis, the TCA cycle, the respiratory chain, NADH shuttles and the pyruvate cycle, as shown in Table 1 and Fig. 1B. Reactions represented by arrows in Fig. 1B are specified in detail in the Supplementary Materials. The molecular components (metabolites and enzymes) of the model are shown in detail in Supplementary Table 1. ATP is a natural point at which to separate these core metabolic processes from downstream processes taking place at or near the cell membrane $\left(\mathrm{K}_{\mathrm{ATP}}\right.$ channels, voltage-gated $\mathrm{Ca}^{2+}$ channels, and insulin granule exocytosis) because it integrates the outputs of the various metabolic pathways.

Three compartments are considered in our model: the mitochondrial matrix, mitochondrial intermembrane space, and the cytoplasm surrounding the mitochondrion. All the enzymatic reactions are represented by rate equations. Cytoplasmic and mitochondrial pools of metabolite are treated separately, as are cytoplasmic and mitochondrial reactions. Table 1 contains details of cytoplasmic and mitochondrial metabolites and associations of the various reactions and metabolites with individual subpathways. The reactions within the mitochondrion are based on the work done by Yugi and Tomita (2004) (see their article for references to original studies). Other reactions (Halestrap 1975; Matsuoka and Srere 1973; Påhlman et al. 2002; Swierczynski 1980) were collected from the SABIO-RK database (http://www.sabio.villa-bosch.de/SABIORK/). The initial metabolite concentrations are taken from the literature. The matrix volume is set at $4.3 \times 10^{-16}$ in correspondence to the Yugi-Tomita model. The volume of cytoplasm is taken to be $6.5 \times 10^{-12} \mathrm{~L}$.

The metabolite and enzyme concentrations, rate equations, and kinetic parameters were formulated and transferred into SBML (Systems Biology Markup language; Hucka et al. 2004) for simulation. Simulations are performed by CVODE solvers (SUNDIALS suite) (Hindmarsh et al. 2005; Serban and Hindmarsh 2005) with Matlab (Mathworks, Natick, MA) and the Systems Biology Toolbox (Schmidt and Jirstrand 2006).

Because our initial set of parameters came from different sources, we needed to make them all consistent in this system. To do this we assumed the parameters within one reaction to be constant, then we scaled the rate equations appropriately.

\section{Network analysis}

Choke point analysis was performed using the Pathway Hunter Tool (Rahman and Schomburg 2006) to identify the enzymes or reactions that are crucial for the network. A choke point is defined as an enzyme or reaction that uniquely consumes and/or produces a certain metabolite.

Normally, the simulation results of a large-scale model depend on various parameters, through the parameters of rate equations and the initial conditions. Sensitivity analysis measures the sensitivity of the simulation results with respect to the model parameters to estimate which parameters are most influential in affecting the behavior of the simulation. The solution of sensitivity with respect to the model parameter $p_{i}$ is defined as the vector

$s_{i}(t)=\frac{\partial m(t)}{\partial p_{i}}$

and satisfies the following equations:

$s_{i}=\frac{\partial f}{\partial m} s_{i}+\frac{\partial f}{\partial p_{i}}$

$s_{i}\left(t_{0}\right)=\frac{\partial m_{0}(p)}{\partial p_{i}}$

The outputs of the above partial equations are parameter sensitivity trajectories (versus time). For each trajectory, the bigger the changes the more the model simulation results are sensitive to the related $p_{i}$ and vice versa. We calculated the sensitivity trajectories and scaled them into 0-1 (shown as a matrix) to show parameter sensitivities versus simulation output of the model. Sensitivity analysis was performed with the SBaddon package v1.1 and the Systems Biology Toolbox (Schmidt and Jirstrand 2006) for 
Table 1 Membership of subpathways in the model

\begin{tabular}{|c|c|c|c|}
\hline Subpathway & Reactions & Enzymes & Metabolites \\
\hline \multirow{12}{*}{$\begin{array}{l}\text { Glycolysis } \\
\quad \text { (cytoplasmic) }\end{array}$} & \multirow[t]{12}{*}{ v1-v7 } & Glucokinase (GK) & Glucose (GLC) \\
\hline & & 6-Phosphofructokinase (PFK) & Fructose-6-phosphate (F6P) \\
\hline & & Fructose-bisphosphate aldolase (FBA) & $\mathrm{ADP}(\mathrm{cyt})$ \\
\hline & & Glyceraldehyde 3-phosphate dehydrogenase (GAPD) & $\operatorname{ATP}($ cyt $)$ \\
\hline & & Bisphosphoglycerate phosphatase (PGP) & $\begin{array}{l}\text { Fructose-1,6-bisphosphate } \\
\text { (FBP) }\end{array}$ \\
\hline & & Pyruvate kinase (PK) & $\begin{array}{l}\text { Glyceraldehyde 3-phosphate } \\
\text { (GAP) }\end{array}$ \\
\hline & & \multirow[t]{6}{*}{ Lactate dehydrogenase (LDH) } & $\begin{array}{l}\text { 1,3-bisphospho-D-glycerate } \\
\text { (DPG) }\end{array}$ \\
\hline & & & $\mathrm{NAD}+(\mathrm{cyt})$ \\
\hline & & & NADH(cyt) \\
\hline & & & Phosphoenol pyruvate (PEP) \\
\hline & & & Pyruvate (PYR(cyt)) \\
\hline & & & Lactate (LAC) \\
\hline \multirow{21}{*}{$\begin{array}{l}\text { TCA cycle } \\
\quad \text { (mitochondrial) }\end{array}$} & \multirow[t]{21}{*}{ v9-v12, v14-v18, v23,v36 } & Pyruvate dehydrogenase complex (PDC) & Carbon dioxide $\left(\mathrm{CO}_{2}\right)$ \\
\hline & & Citrate synthase (CS) & Citrate (Cit) \\
\hline & & Aconitase (ACO) & Coenzyme A (CoA) \\
\hline & & Isocitrate dehydrogenase (NAD+) (IDHa) & Oxaloacetate (OXA) \\
\hline & & Phosphate carrier (PiC) & Acetyl_CoA \\
\hline & & Oxoglutarate dehydrogenase complex (OGDC) & Isocitrate (IsoCit) \\
\hline & & Succinyl-CoA synthetase (SCS) & Oxoglutarate (OG) \\
\hline & & Succinate dehydrogenase (SDH) & Phosphate (Pi) \\
\hline & & Fumarase $(\mathrm{FM})$ & Succinyl-CoA (SCoA) \\
\hline & & Malate dehydrogenase (MDH) & Succinate (Suc) \\
\hline & & Aspartate transaminase (AspTA) & Guanosine diphosphate (GDP) \\
\hline & & Nucleoside diphosphate kinase (NDK) & Guanosine triphosphate (GTP) \\
\hline & & Pyruvate carboxylase (PC) & Fumarate (Fum) \\
\hline & & & Ubiquinone (Q) \\
\hline & & & Ubiquinol (QH2) \\
\hline & & & Malate (Mal) \\
\hline & & & Glutamate (Glu) \\
\hline & & & Aspartate (Asp) \\
\hline & & & $\mathrm{ADP}$ \\
\hline & & & ATP \\
\hline & & & PYR \\
\hline \multirow{10}{*}{$\begin{array}{l}\text { Respiratory chain } \\
\text { (mitochondrial) }\end{array}$} & \multirow[t]{10}{*}{ v24-v26, v28 } & Complex-I & NAD+ \\
\hline & & Complex-III & $\mathrm{NADH}$ \\
\hline & & Complex-IV & Q \\
\hline & & Complex-V & $\mathrm{QH} 2$ \\
\hline & & & Ferricytochrome C (Cyt_c2+) \\
\hline & & & Ferrocytochrome C (Cyt_c3+) \\
\hline & & & Water $(\mathrm{H} 2 \mathrm{O})$ \\
\hline & & & $\mathrm{ADP}$ \\
\hline & & & ATP \\
\hline & & & $\mathrm{Pi}$ \\
\hline
\end{tabular}


Table 1 continued

\begin{tabular}{|c|c|c|c|}
\hline Subpathway & Reactions & Enzymes & Metabolites \\
\hline NADH shuttles & $\begin{array}{l}\text { V16-v18, v20-v22, v30-v32, } \\
\text { v34-v35, v37-v38 }\end{array}$ & $\begin{array}{l}\text { FM } \\
\text { Alanine transaminase (AlaTA) } \\
\text { SDH } \\
\text { AspTA } \\
\text { Aspartate/glutamate carrier (AGC) } \\
\text { Oxoglutarate carrier (OGC) } \\
\text { MDH } \\
\text { ETF:Q oxidoreductase (ETF-QO) } \\
\text { Glutathione reductase (GSSGR) } \\
\text { Pyruvate carboxylase (PC) } \\
\text { Glycerol-3-phosphate dehydrogenase } \\
\text { (FAD dependent) (GUT2P) } \\
\text { Glycerol-3-phosphate dehydrogenase (NAD+) (G3PD) }\end{array}$ & $\begin{array}{l}\text { NAD+ } \\
\text { NADH } \\
\text { NAD+(cyt) } \\
\text { NADH(cyt) } \\
\text { OXA } \\
\text { OXA(cyt) } \\
\text { OG } \\
\text { OG(cyt) } \\
\text { Q } \\
\text { QH2 } \\
\text { Mal } \\
\text { Mal(cyt) } \\
\text { Glu } \\
\text { Glu(cyt) } \\
\text { Asp } \\
\text { Asp(cyt) } \\
\text { Electron Transfer Flavoprotein } \\
\quad \text { (oxidized form) (ETF_ox) } \\
\text { Electron Transfer Flavoprotein } \\
\text { (reduced form) (ETF_red) } \\
\text { FAD } \\
\text { FADH2 } \\
\text { Dihydroxyacetone-phosphate } \\
\text { (DHAP) } \\
\text { Glycerol-3-phosphate (G3P) } \\
\text { Fum } \\
\text { Suc }\end{array}$ \\
\hline Pyruvate Cycle & $\begin{array}{l}\text { v10-v12, v18, v27, v29-v31, } \\
\text { v33, v36, v39, v41-v42, v44 }\end{array}$ & $\begin{array}{l}\text { CS } \\
\text { ACO } \\
\text { IDHa } \\
\text { OGC } \\
\text { PC } \\
\text { Citrate carrier (CIC) } \\
\text { Malate dehydrogenase (oxaloacetatedecarboxylating) } \\
\text { (NADP+) (ME) } \\
\text { Cytosolic isocitrate dehydrogenase (NADP+) (IDHc) } \\
\text { MDH }\end{array}$ & $\begin{array}{l}\text { NAD+ } \\
\text { NADH } \\
\text { NAD+(cyt) } \\
\text { NADH(cyt) } \\
\text { Cit } \\
\text { Cit(cyt) } \\
\text { IsoCit } \\
\text { IsoCit(cyt) } \\
\text { Mal } \\
\text { Mal(cyt) } \\
\text { NADP+ } \\
\text { NADPH } \\
\text { NADP+(cyt) } \\
\text { NADPH(cyt) } \\
\text { Acetyl-CoA } \\
\text { Acetyl-CoA(cyt) } \\
\text { CoA } \\
\text { CoA(cyt) }\end{array}$ \\
\hline
\end{tabular}


Table 1 continued

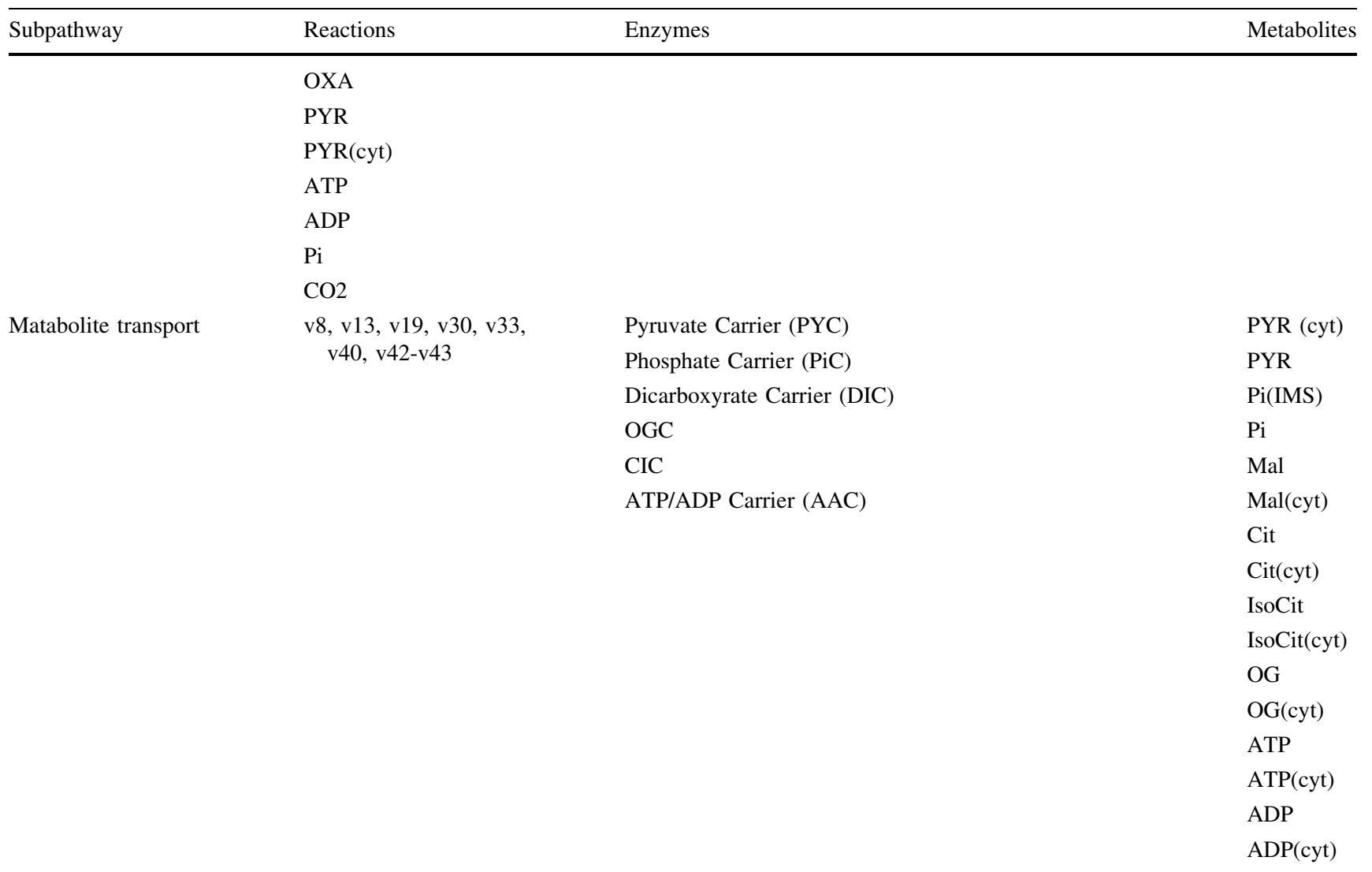

The table provides information on the reactions, enzymes, and metabolites associated with each of the subpathways included in the model. Abbreviations used for enzymes and metabolites elsewhere in the article are given in parentheses. Cytoplasmic and metabolic metabolites are distinguished where relevant.

Matlab, which was also used to perform correlation analysis. Correlation analysis calculates the correlated behavior of parameters in the system during sensitivity analysis and can be taken as an indication of concerted behavior, or constraints, of reactions or reaction parameters.

\section{Results}

Performance analysis

The most basic dynamic property of the GSIS system is oscillation along glycolysis (see Westermark and Lansner 2003) and the concentration relationship between ATP and glucose (Detimary et al. 1996). Figure 2 shows dynamic simulation results of some metabolites in the network. The metabolites along the glycolysis pathway show the desired oscillations. The metabolites outside the glycolysis pathway (e.g., malate and citrate inside the mitochondrion) did not show oscillations. ADP and ATP showed oscillations, as required for stimulation of $\mathrm{Ca}^{2+}$ transport in $\beta$ cells
(Westermark and Lansner 2003). ATP concentration increased with the change of glucose concentration from 0 to $20 \mathrm{mM}$, while ADP concentration decreased. Figure 3 shows the concentration change of ATP with changes of glucose concentration. Consistent with the observations of Detimary et al. (1996), the model predicts ATP levels to increase more than twofold between 0 and $10 \mathrm{mM}$ glucose and then plateau. At $6 \mathrm{mM}$ glucose, the ATP level was 1.78-fold higher than the initial state concentration. ADP progressively decreased to $0 \mathrm{mM}$ as glucose increased from 0 to $6 \mathrm{mM}$ (data not shown). It should be noted that the ATP concentrations predicted by the model represent an upper bound on intracellular ATP concentration because they do not take into account consumption of ATP by $\mathrm{K}_{\mathrm{ATP}}$ channel activity and other cellular processes.

\section{Deletion of malate dehydrogenase}

MacDonald (2002) reported that murine pancreatic islets do not contain detectable levels of malic enzyme (malate dehydrogenase; ME), whereas human and rat islets do. We 

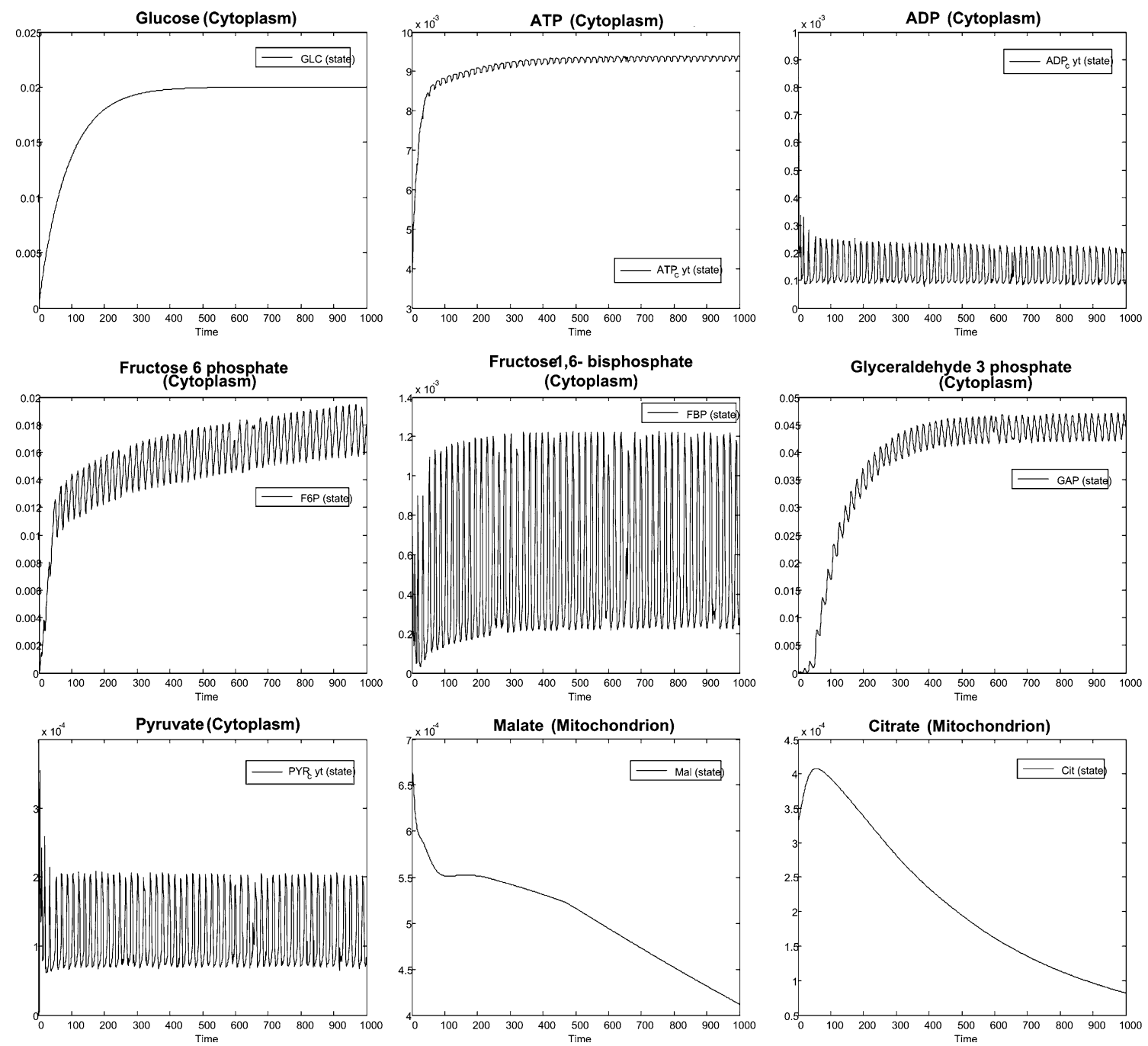

Fig. 2 Simulation results of the model. Plots of the variation in concentration of selected metabolites from a starting state with successive cycles of the model. Concentrations are represented as molar concentrations. The $x$ axis represents individual sequential states of the model

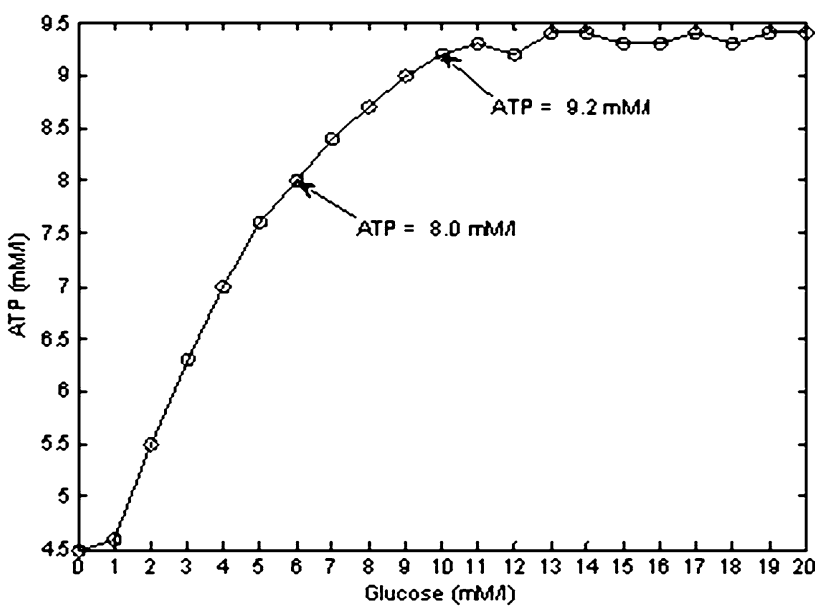

Fig. 3 Relationship of ATP with glucose. The graph shows the effect of varying input glucose concentration on the final ATP concentration of the model after 500 cycles of modeling therefore simulated the effect of removing ME from the model. The model showed a $7.5 \%$ increase in cytoplasmic malate concentration and a $9.6 \%$ decrease in pyruvate on deletion of ME, as well as small effects on the concentrations of some other metabolites (of the order of $1 \%-2 \%$ ). However the resultant ATP concentration was not significantly altered. The oscillatory behavior of the model was also unaffected.

Network analysis

\section{Choke point analysis}

A choke point is defined as a reaction that uniquely consumes and/or produces a certain metabolite. Choke points are critical points in networks (Yeh et al. 2004). In our network inactivation of choke points may lead to a failure to produce or consume particular metabolites that could cause serious problems for ATP production. To detect choke points we input the EC numbers of all enzymes in our 


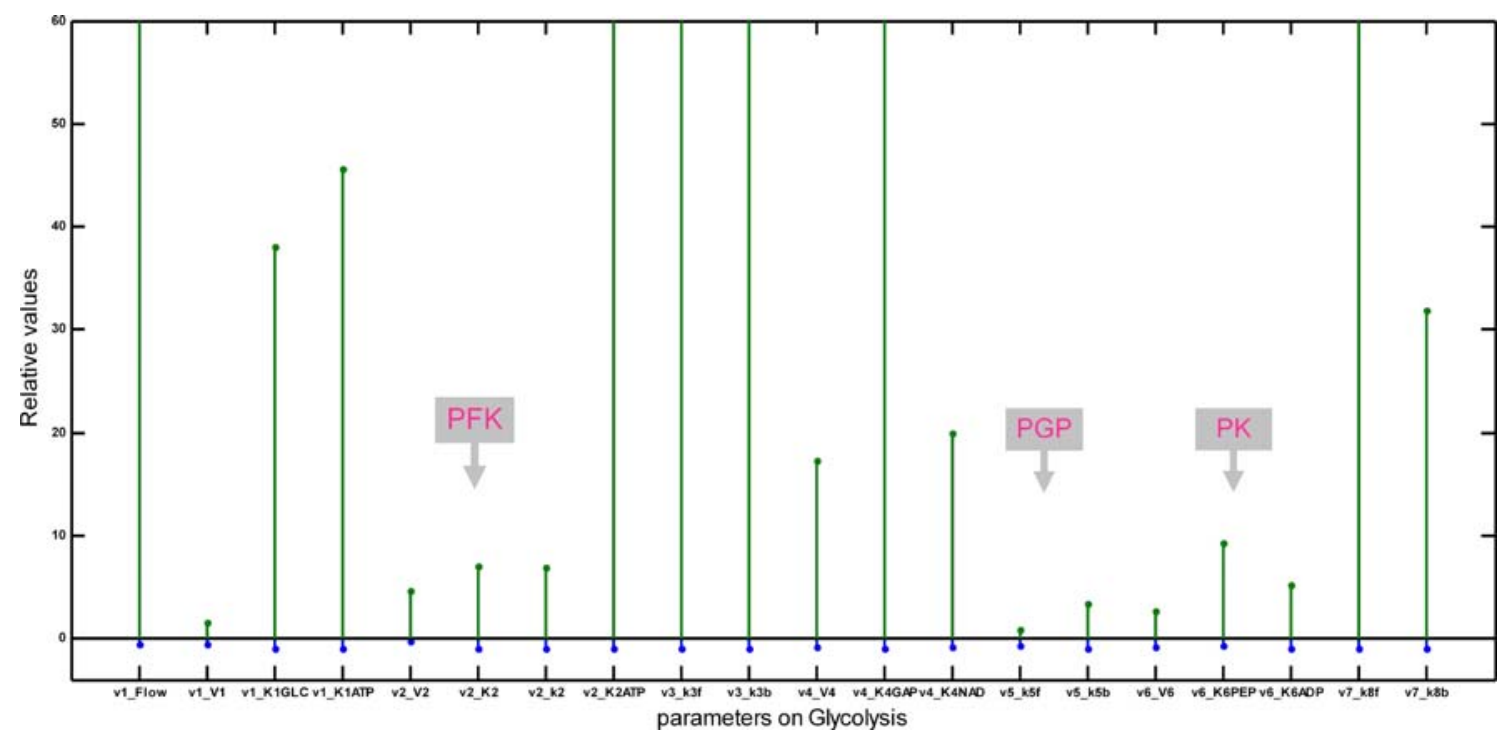

Fig. 4 Parameter space for oscillations (glycolysis pathway). The plot represents ranges of individual parameters (represented as relative values compared with those presented in Supplementary

network into the Pathway Hunter Tool (PHT; Rahman and Schomburg 2006). Six enzymes were identified as choke points: GK (E.C.2.7.1.2; glucokinase), PFK (E.C.2.7.1.11; 6-phosphofructokinase), FBA (E.C. 4.1.2.13; fructose bisphosphate aldolase), PGP (E.C.5.4.2.1/5.4.2.4; bisphosphoglycerate phosphatase), PK (E.C.2.7.1.40; pyruvate kinase), and NDK (E.C.2.7.4.6; nucleoside diphosphate kinase). Five of these are in the glycolysis pathway; we therefore conclude that glycolysis is the most critical subpathway for the functioning of the network.

\section{Parameter space analysis}

Because the glycolysis pathway is the critical pathway in our system and shows oscillations, we calculated the parameter ranges for oscillations in the pathway. The results are shown in Fig. 4. The oscillation behavior of the pathway is sensitive to parameters of reactions v2, v5, and v6. Thus, we predict that the enzymes PFK, PGP, and PK have crucial influences on oscillation, whereas reaction v3 catalyzed by FBA is the most robust reaction.

\section{Sensitivity and correlation analysis}

With a detailed biochemically formulated model, it is convenient to analyze the behavior of any metabolite of interest within the system. However, for analysis of the global properties of the system, the model could be simplified. Sensitivity analysis-based methods can be used to identify those parts of the model that are relatively unimportant for the properties of the system, and those parts could be eliminated from the model (Dano et al. 2006).
Table 3) for which the model produced oscillations in the glycolysis pathway. A complete list of modeling parameters with their origins is provided in the Supplementary Materials

Sensitivity analysis also provides an indication of the biological realisticness of the model. We therefore performed sensitivity analysis of the parameters along each subpathway using SBaddon and the Systems Biology Toolbox (Schmidt and Jirstrand 2006). Figure 5A shows the analysis results for the glycolysis pathway. The rest of the results are shown in the Supplementary Materials. We can see that along the glycolysis pathway parameters in reactions $\mathrm{v} 1, \mathrm{v} 2, \mathrm{v} 6$, and $\mathrm{v} 8$ have the most effect on the state variables. Three reactions in the TCA cycle also showed a high level of sensitivity: v9, v14, and v17. The other subpathways in the model showed less sensitivity to parameter changes than glycolysis. Typically, any sensitivity observed lay at the beginning of a particular subpathway. Thus, sensitivity was observed at reaction $\mathrm{v} 9$ at the beginning of the TCA cycle (from the perspective of incoming pyruvate), at reaction $\mathrm{v} 17$ in the NADH: malate aspartate shuttle, which is the reaction consuming incoming malate, at reaction $\mathrm{v} 24$ in the NADH:glycerophosphate shuttle, which is the first step of the respiratory chain, and at reactions v41 and v44 of the pyruvate cycle.

Correlation analysis is based on the output from sensitivity analysis and represents concerted behavior of parameters in the system. Correlation analysis of glycolysis (Fig. 5B) shows strong interreaction correlations between most of the reactions in the pathway with the exception of reaction $\mathrm{v} 8$ (pyruvate transport across the mitochondrial membrane), which showed only self-correlation. Strong cross-correlation was also observed in the pyruvate cycle. In the other subpathways, particularly the TCA cycle and NADH:malate aspartate shuttle, little evidence of correlation was seen between reactions. However, correlation was 
Fig. 5 A Sensitivity analysis. Individual parameters from reactions in the pathway are arranged vertically and metabolites are arranged horizontally. Sensitivity values on a scale from 0 to 1 are plotted as brown scale boxes at the intersections of the respective columns and rows. High-sensitivity interactions are white, as indicated by the scale on the right. Membership of subpathways and intracellular locations are indicated by bars below the figure. B Correlation analysis. In this case parameters are arranged both vertically and horizontally in the same order so that the diagonal represents self-correlation. High correlations are represented by light browns
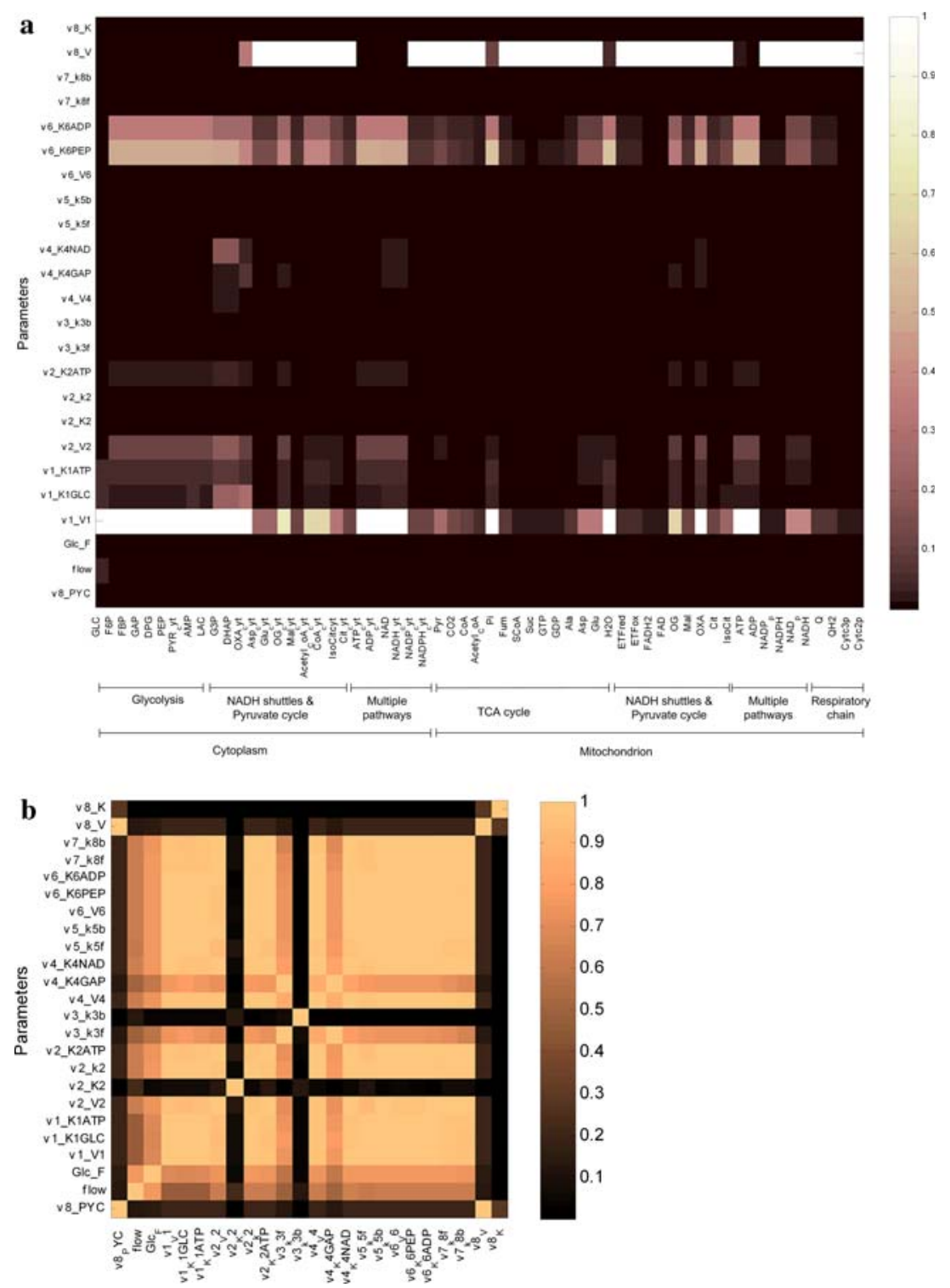

seen only in the NADH:glycerophosphate shuttle between reactions v28, v40, and $\mathrm{v} 43$ (import of ADP into the mitochondrion, production of ATP by complex V, and its export) and reactions $v 38$ (the start of the NADH:glycerophosphate shuttle, catalyzed by G3PD [glycerol-3phosphate dehydrogenase]) and v4 (catalyzed by GAPD [glyceraldehyde-3-phosphate dehydrogenase]).

\section{Discussion}

In this article we describe a mathematical model of the core dynamics of the glucose-stimulated insulin secretion (GSIS) pathway of pancreatic $\beta$ cells. This model draws on a body of previous work on the modeling of the glycolysis pathway (Nielsen et al. 1998) and mitochondrial metabolism (Yugi and Tomita 2004 and references therein) but brings in additional features, notably NADH shuttles and the pyruvate cycle (Eto et al. 1999; Newgard et al. 2002; Ronnebaum et al. 2006; Rubi et al. 2004), to provide a more comprehensive view than has previously been available. The model calculates the changes over time in the concentration of 59 metabolites. We have assessed the behavior of the model with respect to two core properties of the GSIS system: (1) the relationship between ATP output and glucose input concentrations and (2) oscillations of metabolite concentration in the glycolysis pathway. By measuring the nucleotide content of islets isolated from NMR1 mice, Detimary et al. (1996) showed a twofold increase in ATP content between 0 and $10 \mathrm{mM}$ starting glucose concentration, with a subsequent flattening off of the rate of increase. Our model shows the same pattern of ATP concentration change (Fig. 3). Oscillations in the glycolysis pathway are a well-established feature of the system (Westermark and Lansner 2003) and are observed 
in our model for a number of metabolites in this pathway but not for metabolites outside glycolysis (Fig. 2). Metabolites showing oscillatory behavior in our simulations do include ATP and ADP, which have been shown to show coordinated oscillations with glucose-6-phosphate concentration, calcium concentration, and insulin secretion (Deeney et al. 2001; Nilsson et al. 1996).

Figure 4 summarizes investigations designed to map the parameter ranges within which oscillations in the glycolysis pathway are observed in our model. This study identifies the reactions catalyzed by PFK, PGP, and PK (reactions v2, v5, and v6 in Fig. 1B) as being particularly sensitive to parameter changes, while reaction $v 3$ was particularly insensitive to parameter changes. PFK was previously identified as being central to the oscillatory behavior of this pathway and in particular for modulating the frequency of oscillations (Westermark and Lansner 2003), but roles for PGP and PK are not well established and merit further investigation.

We also investigated the global properties of our system. Choke point analysis (Rahman and Schomburg 2006; Yeh et al. 2004) identifies reactions that either uniquely consume a specific substrate or uniquely produce a specific product. These are suggested to be particularly sensitive points in metabolic networks (Yeh et al. 2004). We identified six enzymes-GK, PFK, FBA, PGP, PK, and NDK - as choke points. Five of these are in the glycolysis pathway, the exception being NDK, suggesting that defects in the glycolytic pathways are likely to be particularly deleterious to insulin secretion. Intriguingly, the first of these enzymes, GK, has been found to be mutated in Maturity-Onset Diabetes of the Young, Type II (MODY 2) (Vionnet et al. 1992). This raises the possibility that choke point analysis of particular systems may help identify candidate genes.

Sensitivity analysis of the glycolysis pathway (Fig. 5A) indicates that four reactions (v1, v2, v6, and v8) are sensitive to changes in parameter values. Three of the five choke points in the glycolysis pathway are also identified by sensitivity analysis as important. Reaction v1 is catalyzed by GK, which has been argued to be the primary control point of glycolysis, i.e., it controls the flux through the pathway (Matschinsky et al. 1998; Sweet and Matschinsky 1995; Wang and Iynedjian 1997). V2 is the PFK reaction discussed above, while v6 is the $\mathrm{PK}$ reaction, which is also a choke point on the glycolytic pathway. V8 is the transport of pyruvate into the mitochondrion, whose effects are notably not correlated with the rest of the glycolytic pathway. Outside the glycolytic pathway the model is relatively resistant to perturbation. However, it does show significant sensitivity in reactions $\mathrm{v} 9$ of the TCA cycle (the first step after pyruvate import), v17 of the NADH:malate aspartate shuttle (the first reaction con- suming imported malate), v24 of the NADH:glycerophosphate shuttle (first reaction of the respiratory chain), and v41 and v44 of the pyruvate cycle. Therefore, these may be important control points in the network.

Taken together, these properties of the GSIS core model are consistent with previous experimental and modeling results. It should be emphasized, however, that the concentrations of metabolites that our model predicts have not been tested against experimental evidence. In addition, the parameters of our model, although based on published values and producing acceptable qualitative behavior, have not been optimized as a concerted set. That is, although we have considered the effects of altering individual parameters on the behavior of the system, we have not explored parameter space in a consistent way to estimate an optimal set of values or range of values (e.g., Liebermeister and Klipp 2005). To do this we will require a set of experimental measurements tailored to the optimization of the model. The value of generating such a data set will lie in testing our ability to test the relationships between measured metabolite concentrations and in the model optimization per se.

The utility of this model is expected to lie in its ability to aid our understanding of processes leading to type II diabetes (although it may also have other uses). To do this we will need to extend it to include additional processes, most notably the generation and detoxification of reactive oxygen species, which has recently been implicated in a mouse model of type II diabetes (Freeman et al. 2006a, 2006b), and downstream processes leading to the transport of calcium in response to fluctuations in ATP concentration and the eventual export of insulin.

\section{Comparative aspects}

An important consideration of future analysis will be the comparative aspects of the model. There are two significant issues. First, the structure and parameterization of the current model draw from a number of different studies that have been performed either under different conditions or in different species. Our aim is to build a model that represents the situation in mouse pancreatic $\beta$ cells rather than a generic model. This will require optimization of parameters against experimentally determined concentrations of metabolites under different glucose concentrations and in the absence of specific enzyme activities. This optimization will bear on our second major issue, which is the ability to assess the utility of particular mouse models in the human context. The use of the mouse as a model of human disease is predicated on the assumption that it represents a good approximation of the human state. This assumption is usually based on the relatively close phylogenetic and sequence relationship between humans and mice, but sys- 
tematic study of similarities and differences is lacking. Studies of this kind will be important from an evolutionary perspective, i.e., identifying the kinds of changes that can take place in core parts of metabolism as well as aiding in the understanding of human disease states. We can foresee a range of potential studies that could increase an understanding of this question. These could range from studies of the parts list (are all the necessary genes present in both species? are there gene duplications or deletions in one or another species? what are the patterns of sequence conservation?), to studies of gene expression, splice variation, protein-protein interaction, and protein modification, through to physiologic comparisons of the properties of the systems, including metabolomics, and changes (or otherwise) in the underlying parameters.

Interestingly, we were able to model the effect of one known interspecies difference in this study: the absence of malate dehydrogenase (ME) from mouse (but not rat) pancreatic islets (MacDonald 2002). Deleting ME from our model increased the cytoplasmic malate concentration, as might have been expected, and decreased the pyruvate concentration, but it had no significant effect on ATP concentration or oscillatory behavior. This illustrates the robustness of the model and also how it can serve as the basis for analyses of interspecies differences.

Acknowledgments The authors thank Frances Ashcroft, Andrei Tarasov, and Michael Duchen for discussions. They also thank an anonymous referee for pointing out the absence of malic enzyme activity in mouse pancreatic $\beta$ cells. This work was supported by ENFIN, a Network of Excellence funded by the European Commission within its FP6 Programme, under the thematic area "Life sciences, genomics and biotechnology for health," contract number LSHG-CT-2005-518254.

\section{References}

Ashcroft F, Rorsman P (2004) Type 2 diabetes mellitus: not quite exciting enough? Hum Mol Genet 13:R21-R31

Bertram R, Gram Pedersen M, Luciani DS, Sherman A (2006) A simplified model for mitochondrial ATP production. J Theor Biol 243:575-586

Chassagnole C, Noisommit-Rizzi N, Schmid JW, Mauch K, Reuss M (2002) Dynamic modeling of the central carbon metabolism of Escherichia coli. Biotechnol Bioeng 79:53-73

Dano S, Madsen MF, Schmidt H, Cedersund G (2006) Reduction of a biochemical model with preservation of its basic dynamic properties. FEBS J 273:4862-4877

Deeney JT, Kohler M, Kubik K, Brown G, Schultz V, et al. (2001) Glucose-induced metabolic oscillations parallel those of $\mathrm{Ca}(2+)$ and insulin release in clonal insulin-secreting cells. A multiwell approach to oscillatory cell behavior. J Biol Chem 276:3694636950

Detimary P, Van den Berghe G, Henquin JC (1996) Concentration dependence and time course of the effects of glucose on adenine and guanine nucleotides in mouse pancreatic islets. $\mathrm{J}$ Biol Chem 271:20559-20565
Eto K, Tsubamoto Y, Terauchi Y, Sugiyama T, Kishimoto T, et al. (1999) Role of NADH shuttle system in glucose-induced activation of mitochondrial metabolism and insulin secretion. Science 283:981-985

Freeman H, Cox RD (2006) Type-2 diabetes: a cocktail of genetic discovery. Hum Mol Genet 15:R202-R209

Freeman H, Shimomura K, Horner E, Cox RD, Ashcroft FM (2006a) Nicotinamide nucleotide transhydrogenase: a key role in insulin secretion. Cell Metab 3:35-45

Freeman HC, Hugill A, Dear NT, Ashcroft FM, Cox RD (2006b) Deletion of nicotinamide nucleotide transhydrogenase: a new quantitive trait locus accounting for glucose intolerance in C57BL/6J mice. Diabetes 55:2153-2156

Halestrap AP (1975) The mitochondrial pyruvate carrier. Kinetics and specificity for substrates and inhibitors. Biochem $\mathrm{J}$ 148:85-96

Hindmarsh AC, Brown PN, Grant KE, Lee SL, Serban R, et al. (2005) SUNDIALS: Suite of Nonlinear and Differential/Algebraic Equation Solvers. ACM Trans Math Software 31:363-396

Hoefnagel MH, Starrenburg MJ, Martens DE, Hugenholtz J, Kleerebezem M, et al. (2002) Metabolic engineering of lactic acid bacteria, the combined approach: kinetic modelling, metabolic control and experimental analysis. Microbiology 148:1003-1013

Hucka M, Finney A, Bornstein BJ, Keating SM, Shapiro BE, et al. (2004) Evolving a lingua franca and associated software infrastructure for computational systems biology: the Systems Biology Markup Language (SBML) project. Syst Biol 1:4153

Liebermeister W, Klipp E (2005) Biochemical networks with uncertain parameters. Syst Biol 152:97-107

MacDonald MJ (2002) Differences between mouse and rat pancreatic islets: succinate responsiveness, malic enzyme, and anaplerosis. Am J Physiol Endocrinol Metab 283:E302-E310

Magnus G, Keizer J (1997) Minimal model of beta-cell mitochondrial $\mathrm{Ca} 2+$ handling. Am J Physiol 273:C717-C733

Magnus G, Keizer J (1998a) Model of beta-cell mitochondrial calcium handling and electrical activity. II. Mitochondrial variables. Am J Physiol 274:C1174-1184

Magnus G, Keizer J (1998b) Model of beta-cell mitochondrial calcium handling and electrical activity. I. Cytoplasmic variables. Am J Physiol 274:C1158-C1173

Matschinsky FM, Glaser B, Magnuson MA (1998) Pancreatic betacell glucokinase: closing the gap between theoretical concepts and experimental realities. Diabetes 47:307-315

Matsuoka Y, Srere PA (1973) Kinetic studies of citrate synthase from rat kidney and rat brain. J Biol Chem 248:8022-8030

McCarthy MI (2004) Progress in defining the molecular basis of type 2 diabetes mellitus through susceptibility-gene identification. Hum Mol Genet 13:R33-R41

Newgard CB, Lu D, Jensen MV, Schissler J, Boucher A, et al. (2002) Stimulus/secretion coupling factors in glucose-stimulated insulin secretion: insights gained from a multidisciplinary approach. Diabetes 51:S389-S393

Nielsen K, Sørensen PG, Hynne F, Busse HG (1998) Sustained oscillations in glycolysis: An experimental and theoretical study of chaotic and complex periodic behavior and of quenching of simple oscillations. Biophys Chem 72:49-62

Nilsson T, Schultz V, Berggren PO, Corkey BE, Tornheim K (1996) Temporal patterns of changes in ATP/ADP ratio, glucose 6phosphate and cytoplasmic free $\mathrm{Ca} 2+$ in glucose-stimulated pancreatic beta-cells. Biochem J 314:91-94

Påhlman I-L, Larsson C, Avéret N, Bunoust O, Boubeker S, et al. (2002) Kinetic regulation of the mitochondrial glycerol-3phosphate dehydrogenase by the external NADH dehydrogenase in Saccharomyces cerevisiae. J Biol Chem 277: 27991-27995 
Rahman SA, Schomburg D (2006) Observing local and global properties of metabolic pathways: 'load points' and 'choke points' in the metabolic networks. Bioinformatics 22:17671774

Ronnebaum SM, Ilkayeva O, Burgess SC, Joseph JW, Lu D, et al. (2006) A pyruvate cycling pathway involving cytosolic NADPdependent isocitrate dehydrogenase regulates glucose-stimulated insulin secretion. J Biol Chem 281:30593-30602

Rubi B, del Arco A, Bartley C, Satrustegui J, Maechler P (2004) The malate-aspartate NADH shuttle member Aralar1 determines glucose metabolic fate, mitochondrial activity, and insulin secretion in beta cells. J Biol Chem 279:55659-55666

Schmidt H, Jirstrand M (2006) Systems Biology Toolbox for MATLAB: a computational platform for research in systems biology. Bioinformatics 22:514-515

Serban R, Hindmarsh AC (2005) CVODES: the Sensitivity-Enabled ODE Solver in SUNDIALS. In Proceedings of IDETC/CIE 2005, Long Beach, CA, American Society of Mechanical Engineers, New York, pp DETC2005-85597

Sweet IR, Matschinsky FM (1995) Mathematical model of beta-cell glucose metabolism and insulin release. I. Glucokinase as glucosensor hypothesis. Am J Physiol 268:E775-E788

Swierczynski J (1980) Purification and some properties of extramitochondrial malic enzyme from rat skeletal muscle. Biochim Biophys Acta 616:10-21
Topp B, Promislow K, deVries G, Miura RM, Finegood DT (2000) A model of beta-cell mass, insulin, and glucose kinetics: pathways to diabetes. J Theor Biol 206:605-619

Varner JD (2000) Large-scale prediction of phenotype: concept. Biotechnol Bioeng 69:664-678

Vionnet N, Stoffel M, Takeda J, Yasuda K, Bell GI, et al. (1992) Nonsense mutation in the glucokinase gene causes early-onset non-insulin-dependent diabetes mellitus. Nature 356:721-722

Wang H, Iynedjian PB (1997) Modulation of glucose responsiveness of insulinoma beta-cells by graded overexpression of glucokinase. Proc Natl Acad Sci U S A 94:4372-4377

Westermark PO, Lansner A (2003) A model of phosphofructokinase and glycolytic oscillations in the pancreatic beta-cell. Biophys $\mathrm{J}$ 85:126-139

Westermark PO, Hellgren Kotaleski J, Bjorklund A, Grill V, Lansner A (2006) A mathematical model of the mitochondrial NADH shuttles and anaplerosis in the pancreatic beta-cell. Am J Physiol Endocrinol Metab 292:E373-E393

Yeh I, Hanekamp T, Tsoka S, Karp PD, Altman RB (2004) Computational analysis of Plasmodium falciparum metabolism: organizing genomic information to facilitate drug discovery. Genome Res 14:917-924

Yugi K, Tomita M (2004) A general computational model of mitochondrial metabolism in a whole organelle scale. Bioinformatics 20:1795-1796 